\title{
Conformation and Surface Properties of Deamidated Gluten
}

\author{
Naotoshi Matsudomi, Akio Kato and Kunihiko Kobayashi \\ Department of Agricultural Chemistry, Yamaguchi University, \\ Yamaguchi 753, Japan \\ Received November 13, 1981
}

\begin{abstract}
The surface properties of deamidated gluten were investigated with respect to their conformational changes. The helix content of gluten decreased curvilinearly with its decrease of deamidation. The surface tension decreased in proportion to the degree of deamidation. On the other hand, the surface hydrophobicity of gluten increased remarkably in proportion to the degree of deamidation. The emulsifying properties of gluten were improved greatly by deamidation, correlating linearly with the surface hydrophobicity. From these results, the relationships between the conformational changes and functional properties of deamidated gluten are discussed.
\end{abstract}

In the previous paper, ${ }^{1)}$ we reported that the functional properties of gluten, including solubility, emulsifying properties and foaming properties, were greatly improved by mild acid hydrolysis. It was also suggested that the improved functional properties were mainly due to a deamidation process and not to a cleavage of peptide linkages in the gluten molecules. In addition, it was assumed that deamidated gluten increased the functional properties of gluten because this deamidation might induce conformational changes by increasing the electrostatic repulsion and decreasing the hydrogen bonding.

It has been recently reported that the surface structure of proteins plays an important role in the functional properties of food protein. Some investigators ${ }^{2} \sim 5$ ) have reported that surface hydrophobicity showed a significant correlation with the emulsifying properties of the various proteins. Therefore, it can be presumed that the functional properties improved by deamidation may be due to an increase in the effective hydrophobicity of gluten. However, little is known about the surface structure of gluten. Therefore, further investigation on the surface structure of gluten needed to be done to correlate protein functionality with conformation. This paper describes the effect of conformational changes on the surface properties of deamidated gluten.

\section{MATERIALS AND METHODS}

Source and preparation of gluten. Wheat flour was obtained from Torigoe Flour Milling Co. Ltd. (Japan). Insoluble wheat protein complex, gluten, was prepared from a flour dough washed until the washings were free from soluble protein. The gluten ball thus obtained was dialyzed against distilled water and then freeze-dried.

Preparation of deamidated gluten samples. Deamidated gluten was prepared using a slightly modified version of method. ${ }^{1)}$ A $5 \%$ gluten suspension in $0.05 \mathrm{~N}$ or $0.1 \mathrm{~N} \mathrm{HCl}$ was heated at $120^{\circ} \mathrm{C}$ for different time intervals. The acidtreated gluten was adjusted to $\mathrm{pH} 7$ with $1 \mathrm{~N} \mathrm{NaOH}$. The gluten solution was dialyzed exhaustively against distilled water and then freeze-dried.

Chemical analysis. Amide nitrogen was determined as distillable ammonia after refluxing the deamidated gluten in $2 \mathrm{~N} \mathrm{HCl}$ for $3 \mathrm{~h}^{6)}$ Protein concentration was determined by the method of Lowry et al., ${ }^{7)}$ using bovine serum albumin as a standard. SH- and SS-groups in the gluten protein were determined according to method of Beveridge et al.,9) using Ellman's reagent.

Circular dichroism (CD) analysis. $20 \mathrm{mg}$ of the gluten sample was dispersed in $10 \mathrm{ml}$ of $0.1 \mathrm{M}$ Mcllvaine buffer, at pH 3.0 and $\mathrm{pH}$ 8.0.

Each of these gluten suspensions was homogenized lightly in an Ultra Turrax Disperser TP 18 (Hansen \& Co., Ltd.) and then filtered through filter paper (Toyo Roshi, No. $5 B$ ). The soluble protein solution thus obtained was 
used for $\mathrm{CD}$ analysis and the determination of protein concentration. CD was measured on a Jasco Model J-20 C spectropolarimeter under the far-ultraviolet region condition $(200 \sim 300 \mathrm{~nm})$ with a $0.01 \mathrm{~cm}$ path length cell at $25^{\circ} \mathrm{C}$. The helix content was calculated from the mean residue ellipticity at $222 \mathrm{~nm}$, using 110 as the residue

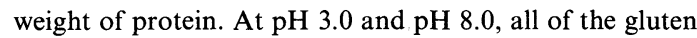
samples had a similar value for helix content.

Determination of surface hydrophobicity. $20 \mathrm{mg}$ of the gluten sample was dispersed in $10 \mathrm{ml}$ of $0.01 \mathrm{M}$ phosphate buffer, $\mathrm{pH}$ 7.4. The soluble protein solution was obtained in a similar manner to that described in the $\mathrm{CD}$ analysis. This solution was used for the determination of surface hydrophobicity, which was determined by the fluorescence technique using cis-parinaric acid. ${ }^{4)}$ The initial slope $\left(S_{0}\right)$, i.e. fluorescence intensity $/ \%$ protein, was calculated from plots of the fluorescence intensity vs. protein concentration.

Measurement of surface properties. Surface tension was measured with a $0.2 \%$ protein solution in a $0.01 \mathrm{M}$ phosphate buffer ( $\mathrm{pH} 7.4$ ) on a Dunoüy Interfacial Tensiometer (Kyowa Kagaku K. K., Japan) at $25^{\circ} \mathrm{C}$.

The emulsifying activity index was determined according to the method of Pearce and Kinsella. ${ }^{8}$ To prepare the emulsions, $1.8 \mathrm{ml}$ of corn oil and $4.2 \mathrm{ml}$ of $0.2 \%$ protein solution in a $0.01 \mathrm{M}$ phosphate buffer $(\mathrm{pH}$ 7.4) were homogenized in a Disperser at $12000 \mathrm{rpm}$ for $1 \mathrm{~min}$ at $25^{\circ} \mathrm{C}$. Emulsion stability was calculated from the following procedure. Aliquots $(100 \mu \mathrm{l})$ of the emulsion obtained above were taken from the bottom of the container after different time intervals $(0,1,2,5,10$ and $30 \mathrm{~min})$ and diluted with $5 \mathrm{ml}$ of $0.1 \%$ SDS solution. The absorbance of the diluted emulsion was then determined at a wave-length of $500 \mathrm{~nm}$ and indicated as a turbidity. The turbidity halftime was calculated from plots of turbidity $v s$. time-course. The emulsion stability was expressed as the value of halftime $\times$ initial turbidity.

\section{RESULTS AND DISCUSSION}

\section{Conformational changes by the deamidation of gluten}

In the previous paper, ${ }^{1)}$ we suggested that acid-modified gluten with less than $40 \%$ deamidation may induce conformational changes without cleavage of the peptide linkages in the gluten molecules. On the other hand, conformational changes due to SS disruption may occur during the acid hydrolysis of gluten. Therefore, the contents of the SH- and SSgroups in the deamidated gluten were measured. As shown in Fig. 1, changes in the SH-

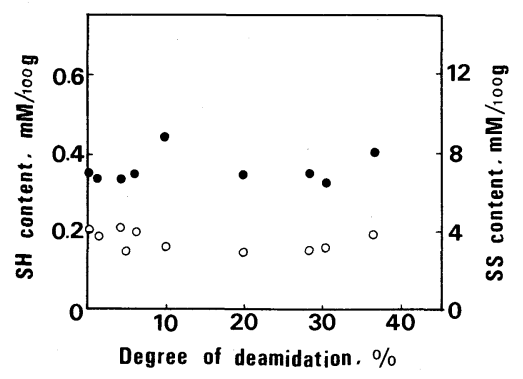

FIG. 1. Changes in SH- and SS-Contents during Deamidation of Gluten.

$\bigcirc, \mathrm{SH}$ group; $\mathrm{SS}$ group.

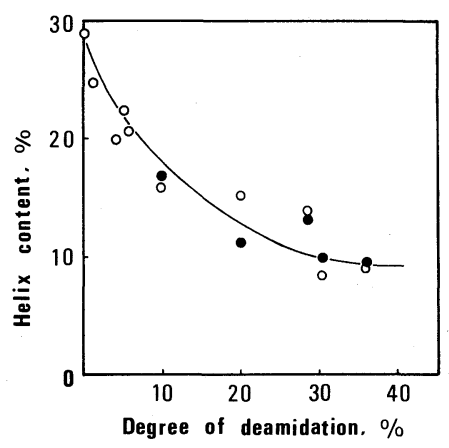

FIG. 2. Relationship between Helix Content and Deamidation of Gluten.

O, determined at $\mathrm{pH} 3.0 ; \bigcirc$, determined at $\mathrm{pH}$ 8.0.

and SS-groups during deamidation of the gluten were nonsignificant, although the possibility of conformational changes due to the SS interchange reaction still exists. In addition, the helix content of the deamidated gluten was measured to elucidate these conformational changes. Figure 2 shows the relationship between the helix content and the degree of deamidation in the gluten. The helix content decreased curvilinearly with the increase of deamidation. This suggests that the conformational changes may be caused mainly by the increased electrostatic repulsion and the decreased hydrogen bonding as a result of deamidation. When the degree of deamidation in gluten became about $40 \%$, the helix content of the gluten decreased to about $10 \%$ from the $30 \%$ of native gluten. However, the helix content was always retained at about $10 \%$ even when the degree of deamidation was over $40 \%$ 
(data not shown). This suggests that a stable structure may partially exist in the gluten molecules. Figure 3 shows the relationship between the surface hydrophobicity and the degree of deamidation in gluten. Surface hy drophobicity increased linearly in proportion to the degree of deamidation and a close correlation $(r=0.97, p<0.01)$ was observed between these two parameters.

This result suggests that the hydrophobic regions are exposed at the molecular surface in proportion to the conformational changes caused by deamidation. Figure 4 shows the relationship between surface tension and the degree of deamidation in gluten. Surface tension decreased linearly in proportion to the deamidation and the correlation coefficient between these parameters was -0.91 and highly significant $(p<0.01)$. This result suggests that the deamidation of gluten cause a pronounced reduction of surface free energy at the interface between air and water, prob-

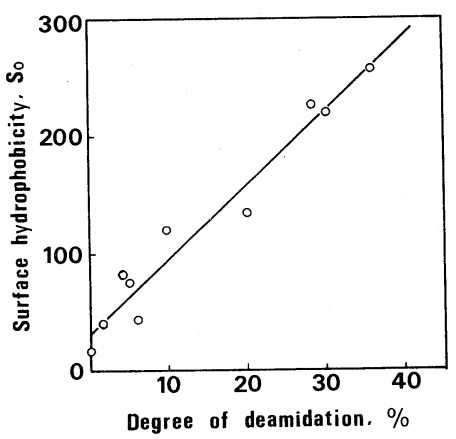

FIG. 3. Relationship between Surface Hydrophobicity and Deamidation of Gluten.

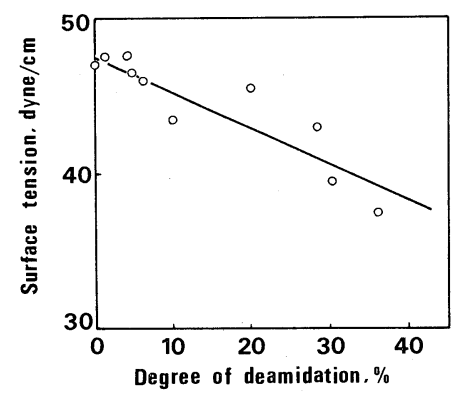

FIG. 4. Relationship between Surface Tension and Deamidation of Gluten. ably due to induction of an amphiphilic nature by the remarkable increase in surface hydrophobicity.

\section{Relationship between surface hydrophobicity} and surface properties

We have already reported that the surface properties of gluten were improved markedly by deamidation. It is probable that the main structural factor governing the functional properties of proteins may be the hydrophobic regions at the protein surface which enhance the interaction between the protein and other molecules, such as lipids. Therefore, the surface properties of deamidated gluten were correlated with the surface hydrophobicity.

The relationships of emulsifying activity and emulsion stability with surface hydrophobicity of the deamidated gluten are shown in Figs. 5

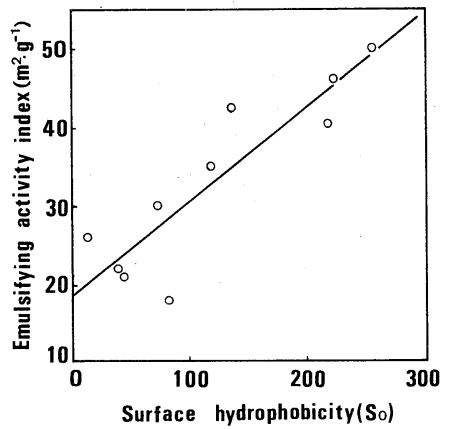

FIG. 5. Relationship between Emulsifying Activity Index and Surface Hydrophobicity of Deamidated Gluten.

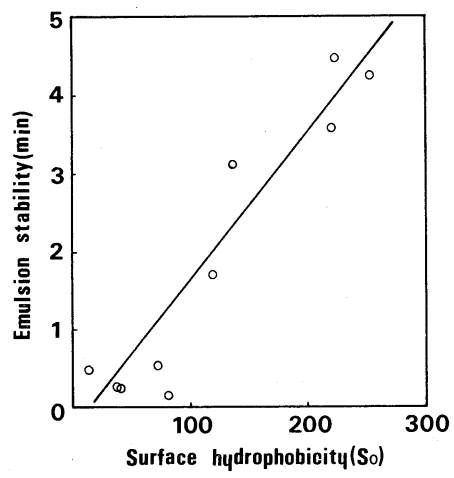

FIG. 6. Relationship between Emulsion Stability and Surface Hydrophobicity of Deamidated Gluten. 


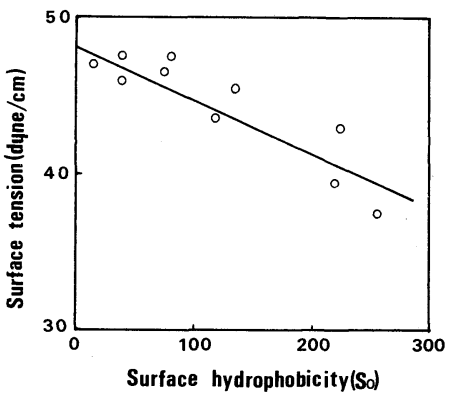

FIG. 7. Relationship between Surface Tension and Surface Hydrophobicity of Deamidated Gluten.

and 6 , respectively. The emulsifying activity index correlated linearly with surface hydrophobicity and the correlation coefficient was 0.87 and highly significant $(p<0.01)$. As shown in Fig. 6, the emulsion stability also correlated linearly with surface hydrophobicity and the correlation coefficient was 0.95 and highly significant $(p<0.01)$. From these results, it was confirmed that the emulsifying properties of gluten were improved greatly by deamidation, correlating linearly with increased surface hydrophobicity. Figure 7 shows the relationship between the surface tension and surface hydrophobicity of deamidated gluten. A good correlation $(r=-0.89$, $p<0.01)$ was observed between these param- eters. Thus, it was elucidated that the surface properties of deamidated gluten were improved, depending mainly on the surface hydrophobicity.

In conclusion, a marked increase in surface hydrophobicity is the main structural factor for an improvement in functional properties by the deamidation of gluten. Induction of an amphiphilic nature, due to an increase in surface hydrophobicity, causes a decrease in free energy at the surface of the deamidated gluten and endows good surface properties.

\section{REFERENCES}

1) N. Matsudomi, S. Kaneko, A. Kato and K. Kobayashi, Nippon Nôgeikagaku Kaishi, 55, 983 (1981).

2) T. Horiuchi, D. Fukushima, H. Sugimoto and T. Hattori, Fd. Chem., 3, 35 (1978).

3) S. Nakai, L. Ho, N. Helbig, A. Kato and M. A. Tung, Can. Inst. Food Sci. Technol. J., 13, 23 (1980).

4) A. Kato and S. Nakai, Biochim. Biophys. Acta, 624, 13 (1980).

5) A. Kato, N. Tsutsui, N. Matsudomi, K. Kobayashi and S. Nakai, Agric. Biol. Chem., 45, 2755 (1981).

6) P. E. Wilcox, Methods Enzymol., 11, 63 (1967).

7) O. H. Lowry, N. J. Rosebrough, A. L. Farr and R. J. Randall, J. Biol. Chem., 193, 265 (1951).

8) K. N. Pearce and J. E. Kinsella, J. Agric. Food Chem., 26, 716 (1978).

9) T. Beveridge, S. J. Toma and S. Nakai, J. Food. Sci., 39, 49 (1974). 